\title{
Real-Time Characterization of Electrospun PVP Nanofibers as Sensitive Layer of a Surface Acoustic Wave Device for Gas Detection
}

\author{
D. Matatagui, ${ }^{1}$ M. J. Fernández, ${ }^{1}$ J. P. Santos, ${ }^{1}$ J. Fontecha, ${ }^{1}$ I. Sayago, ${ }^{1}$ \\ M. C. Horrillo, ${ }^{1}$ I. Gràcia, ${ }^{2}$ and C. Cané ${ }^{2}$ \\ ${ }^{1}$ Instituto de Tecnologías Físicas y de la Información (ITEFI), Consejo Superior de Investigaciones Científicas (CSIC), \\ Serrano 144, 28006 Madrid, Spain \\ ${ }^{2}$ Instituto de Microelectrónica de Barcelona, CSIC, Campus UAB, 08193 Bellaterra, Spain
}

Correspondence should be addressed to J. P. Santos; jp.santos@csic.es

Received 11 October 2013; Revised 21 January 2014; Accepted 23 January 2014; Published 13 March 2014

Academic Editor: Sheng-Joue Young

Copyright (C) 2014 D. Matatagui et al. This is an open access article distributed under the Creative Commons Attribution License, which permits unrestricted use, distribution, and reproduction in any medium, provided the original work is properly cited.

The goal of this work has been to study the polyvinylpyrrolidone (PVP) fibers deposited by means of the electrospinning technique for using as sensitive layer in surface acoustic wave (SAW) sensors to detect volatile organic compounds (VOCs). The electrospinning process of the fibers has been monitored and RF characterized in real time, and it has been shown that the diameters of the fibers depend mainly on two variables: the applied voltage and the distance between the needle and the collector, since all the electrospun fibers have been characterized by a scanning electron microscopy (SEM). Real-time measurement during the fiber coating process has shown that the depth of penetration of mechanical perturbation in the fiber layer has a limit. It has been demonstrated that once this saturation has been reached, the increase of the thickness of the fibers coating does not improve the sensitivity of the sensor. Finally, the parameters used to deposit the electrospun fibers of smaller diameters have been used to deposit fibers on a SAW device to obtain a sensor to measure different concentrations of toluene at room temperature. The present sensor exhibited excellent sensitivity, good linearity and repeatability, and high and fast response to toluene at room temperature.

\section{Introduction}

Classical systems to detect volatile organic compounds (VOCs) as ion mobility spectroscopy [1, 2], chromatographic flame photometry [3], infrared spectrometry [4], Raman spectroscopy [5], and photoionization [6] are used for different purposes, such as detecting food quality, hazardous chemical vapors, chemical warfare agents, potential fires, and environmental pollutants. But its use is limited by factors such as the need for highly qualified personnel, complex heavy and expensive instrumentation or the difficulty and slowness of sample preparation, and the need for complex systems for the analysis of results. Solid-state gas sensors have been studied and used for more than 30 years [7-16] because they are of low cost, work in situ and in real time, with low power consumption, are highly sensitive, and are suitable for portable systems [17].
Among the solid-state gas sensors, the surface acoustic wave (SAW) sensors have received increasing attention due to their fast response time, small size, low cost, very high sensitivity, and their ability to measure in real time and at room temperature $[18,19]$. The SAW devices can be functionalized using a great variety of materials as sensitive layers, polymers usually, deposited on the active area of the SAW device. The sensitive layer then adsorbs volatiles and changes the mass surface density, thus causing a variation in the velocity of the acoustic wave which propagates on the substrate. The variation in the velocity is measured by means of the changes in the resonant frequency of the SAW device. The adsorption is reversible and therefore the system can be reused as many times as necessary.

Polymers are widely used as sensitive layer for many types of sensors. Due to their properties, low cost, and easy preparation, they have been popular candidates for the 
development of high performance SAW chemical sensors. The SAW sensors are often coated with a thin layer of polymer in a rubbery and amorphous state. Therefore, the area in which the reaction between the volatiles and the polymer takes place is the same as the active area of the device. The morphology of the sensing layer plays an important role in the molecular adsorption-desorption process, sensor response, and sensitivity. To take advantage of their larger reaction surface, nanostructured materials can be used as sensitive layer instead of a thin layer because they improve the sensor performance. Therefore, sensitive layers of nanostructured materials could provide higher sensitivity and velocity of reaction with lower insertion losses than the sensitive thin films coated with methods such as drop, airbrush spray, or spinning.

Among the various methods used to prepare nanostructured materials, electrospinning is one of the most convenient options and is especially suitable for the preparation of nanostructured polymer fibers [20-24]. The electrospinning technique is a simple, efficient, and low cost method to deposit polymers in the form of nanofibers to use as sensitive layer for SAW devices [25-28]. It also has the advantage of not requiring the use of coagulation chemistry or high temperatures for the deposition of nanostructures. Electrostatic stretching is an efficient method to make ultrathin fibers ranging in diameters from several micrometers to few nanometers.

In order to create a VOC sensor by means of electrospinning, PVP fibers have been deposited onto the surface of the SAW device as sensitive material and the fibers have been characterized by a SEM detailed study, where the diameters and the fiber distribution were studied. Furthermore, this study provides a novel method for studying the effects of different variables on the morphology of the fibers in the electrospinning process with a SAW oscillator while a high voltage is applied. Among the SAW sensors a Love-wave device has been chosen to develop a gas sensor. The PVP fibers were deposited onto the surface of the Love-wave device and their sensing properties were tested using toluene.

\section{Materials and Methods}

2.1. SAW Device. The SAW devices used in this work were of Love-wave type (Figure 1). They were based on a shear horizontal surface acoustic wave (SH-SAW) propagated on the ST- $x 90^{\circ}$ cut quartz substrate, perpendicular to the $x$ crystallographic axis. This SH-SAW, with a wavelength of $\lambda=28 \mu \mathrm{m}$, was generated and detected by interdigital transducers (IDTs). The IDTs were made using standard lithographic technique depositing an aluminum layer with a thickness of $200 \mathrm{~nm}$ through RF sputtering and forming a delay line (DL). A double electrode structure was repeated 75 times to form each IDT. The spacing, center to center between IDTs (Lcc), was $225 \lambda$ and the acoustic aperture (W) was $75 \lambda$.

Finally, the Love-wave was obtained by guiding the $\mathrm{SH}$ SAW in a film of $\mathrm{SiO}_{2}$ deposited by PECVD. The highest sensitivity was found at a thickness of approximately $3.5 \mu \mathrm{m}$ of

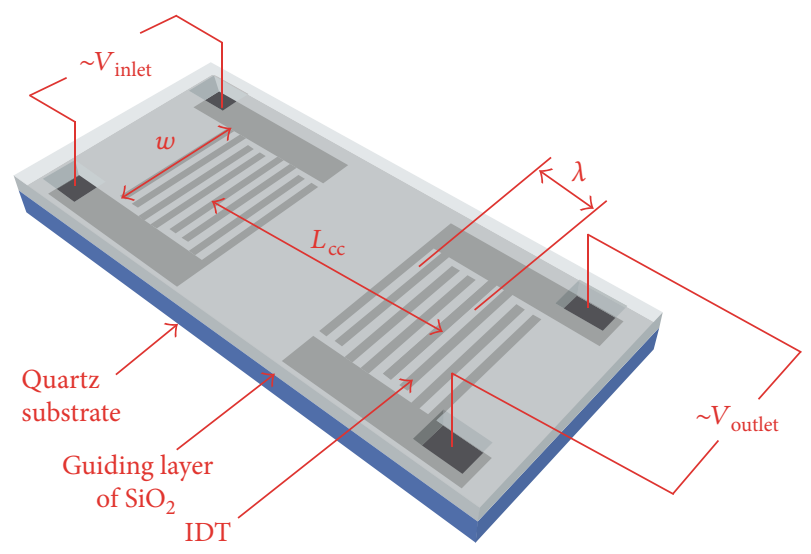

FIGURE 1: 3D scheme representing a Love-wave sensor with two RF ports, layer composition, and geometrical parameters.

$\mathrm{SiO}_{2}$ [29], the synchronous frequency being around $163 \mathrm{MHz}$ [11].

Before and after the electrospinning of the fibers onto the device, the sensors were electrically characterized measuring the frequency response in amplitude and phase by means of a vector network analyzer (Agilent 5070B). Moreover the working frequency of the oscillator was measured through a spectrum analyzer (Agilent 9320A).

2.2. Electrospinning Setup and Process. The starting polymer was Polyvinylpyrrolidone (PVP) (Sigma 81440). It is a water soluble nonconducting polymer which has excellent wetting properties and readily forms films. This makes it good as a coating or an additive to coatings in a wide variety of applications in medicine, pharmacy, cosmetics, and industrial production. It is also used as sensing material for detecting organic vapours in gas sensor. Our solution for electrospinning was prepared by dissolving PVP powder $\left(\mathrm{Mw}=360000 \mathrm{~g} \cdot \mathrm{mol}^{-1}\right)$ in deionized water with a concentration of $25 \%$ and stirring it at room temperature for $4 \mathrm{~h}$.

The polymer solution was loaded into a $10 \mathrm{~mL}$ glass syringe with a $1.1 \mathrm{~mm}$ outer diameter metallic needle which was placed in the syringe pump (NE-1000) where the polymer solution flow rate was selected, being optimized in this work to $5 \mu \mathrm{L} \cdot \mathrm{min}^{-1}$. The needle was connected to a high-voltage power supply and was directed to the grounded collector, which was a round copper plate.

In the electrospinning process, the solution in the syringe is extruded from the needle tip to the collector, where the device is placed. When high voltage is applied between the needle and the collector, an electrostatic force is induced on the droplets of the solution at the needle tip. Electric charges are distributed on the surface of the droplet of solution, resulting in a force of electrostatic repulsion, that together with the Coulomb force due to the applied potential make the shape of the drop results into a conical shape, known as the Taylor cone. When the intensity of electric field reaches a critical value, the electrostatic force overcomes the surface tension of the solution and causes the droplets to stretch, forming thin jets of polymer solution that dry in flight and 


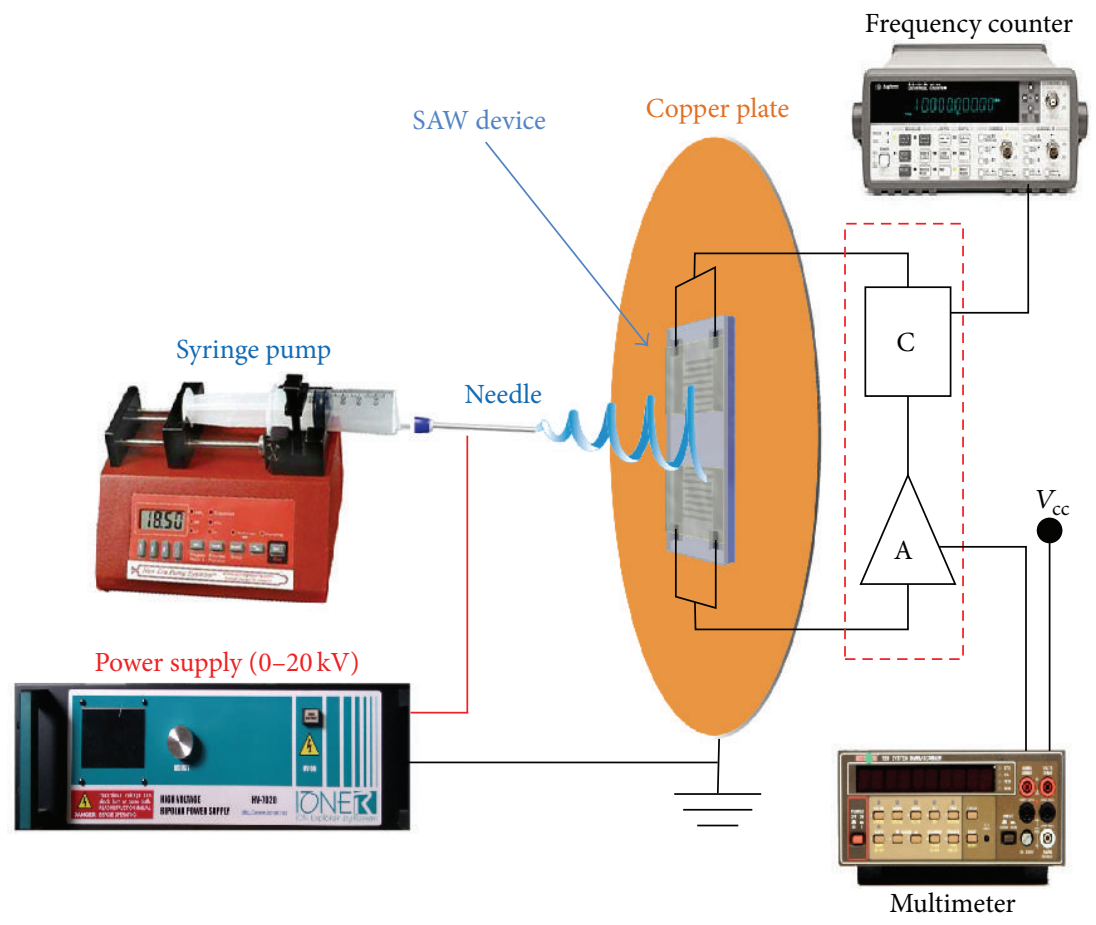

FIGURE 2: Experimental setup for electrospinning and measurement in real time of the deposited fibers.

are deposited onto the collector. If some parameters of electrospinning change, such as the applied voltage, needle-tocollector distance, solution viscosity, or flow rate of solution, the electrospinning process and the morphology of the fibers obtained on the collector may vary. Then a study of the electric field distribution was carried out by means of the simulation software Comsol showing that the field is almost homogeneous in the central part of the collector, where the device is placed, promoting the random distribution of the fibers on the device.

In this work, the SAW device was placed on the collector and the IDTs were covered by a mask of Teflon in order to exclusively deposit fibers onto the space between the IDTs, which is the active zone of the device. The applied voltage and the needle-to-collector distance were then modified and the morphology of the fiber was studied by SEM (Fei Quanta 3D FEG model).

The SAW sensor works in an oscillator system, which includes two amplifiers and a directional coupler. When the device is perturbed due to a change of mass on the surface, the velocity of the surface wave changes; then the oscillating frequency is shifted in order to fulfill the criterion of Barkhausen, which means that the phase of the closed loop is $2 \pi n$ and the gain is $0^{+} \mathrm{dB}$. Furthermore, the attenuation of the device increases when the mass is added on the surface. The amplifier system must then increase the gain in order to maintain the criterion of Barkhausen, mentioned above. Since the amplifier feeding voltage is maintained fixed by the voltage source, the only way to increase its gain is by increasing the feeding current. The increase of the mass on the surface produces an increase of the losses in the
SAW device, which causes an increase of the gain in the amplifier. Therefore, there is an adjustment of the feeding current, which can be measured by means of an amperimeter (Keithley 199). The insertion losses in the SAW device and the feeding current of the amplifier system have been measured in our sensor system for a variety of values and an empirical relation between them has been obtained; that is,

$$
A=C_{1} \cdot I_{F}+C_{2},
$$

where $A$ is the increase of the attenuation of the SAW device in $\mathrm{dB}$ and $I_{F}$ is the feeding current of the amplifier in $\mathrm{mA}$; in our case, $C_{1}=-1.944\left(\mathrm{~dB} \cdot \mathrm{mA}^{-1}\right)$ and $C_{2}=143.7(\mathrm{~dB})$.

A similar relation could be obtained for any SAW sensor working in an oscillating system. In this way the losses in the SAW device can be measured without using an RF measurement system, allowing the monitoring in real time of the losses produced by the increase of the mass on the sensor surface.

The frequency is measured by a frequency counter (Agilent 53131A) through a directional coupler. The frequency counter, the amperimeter, and the data acquisition are controlled in real time through a GPIB protocol by a homemade software specifically developed for this experimental setup. The whole setup can be seen in Figure 2.

2.3. Experimental Setup of the System of Gas Detection. The setup of gas detection consists of the test chamber with the Love wave device, the thermal control system with a Peltier device, the dual channel frequency counter, the flow meters, synthetic air and toluene bottles, and a PC for control and 


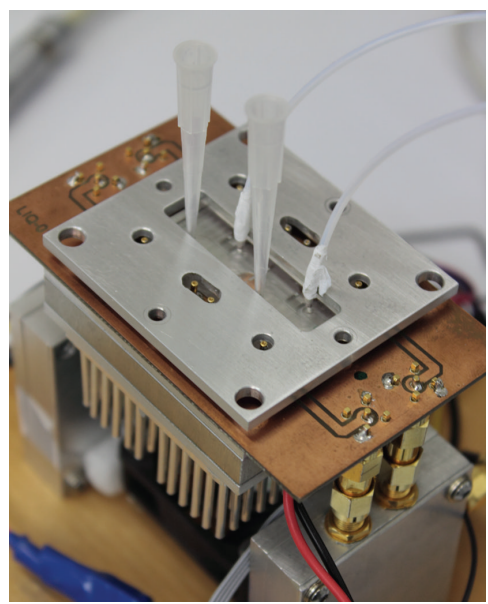

(a)

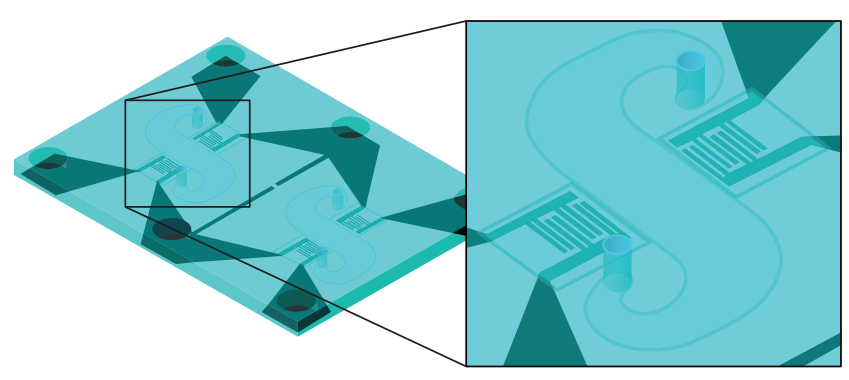

(b)

Figure 3: Gas chamber (a) general view showing the inlet and outlet of gas (b) Love-wave device with two delay lines and a microfluidic chip of PDMS forming the two microchannels.

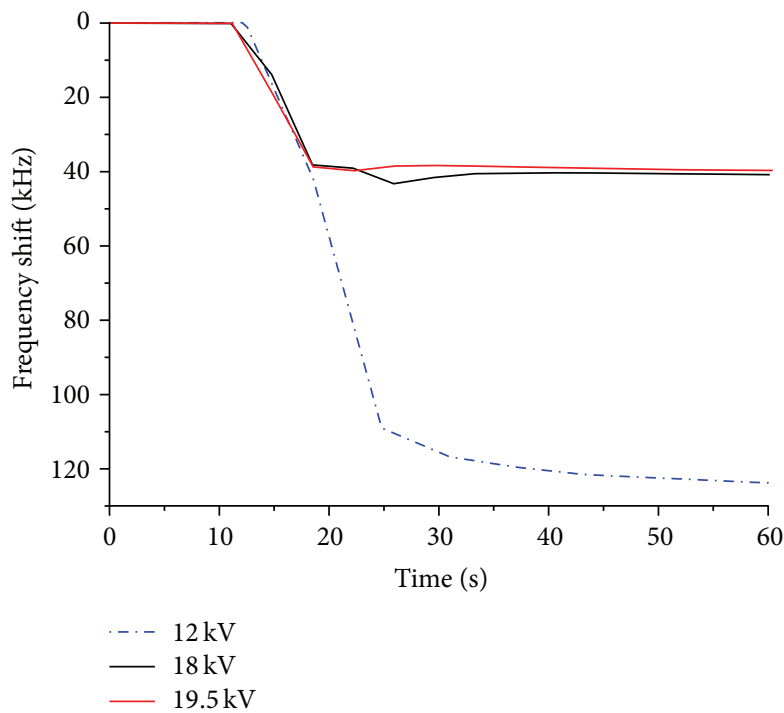

(a)

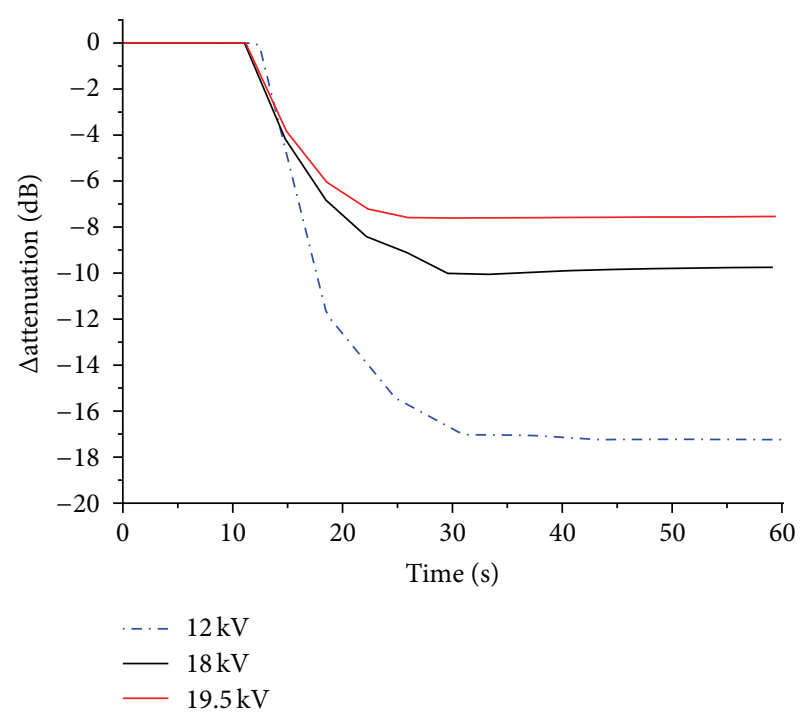

(b)

FIGURE 4: Frequency shift (a) and increasing of the attenuation (b) of the SAW sensor measured in real time due to the PVP electrospun fibers deposited at several applied voltages for a needle-to-collector distance of $11 \mathrm{~cm}$.

data acquisition in real time. The system has been described elsewhere [11]. In order to use a low flow of sample gas, a gas chamber was made based on microchannels in the following way. A PDMS chip was made with a mold of SU-8 with the microchannel shape. The negative photoresist SU-8 was spun on a clean wafer with a thickness of $150 \mu \mathrm{m}$, exposed to an optical lithography process and then baked to obtain the master. The silicone base and curing agent were mixed at a weight ratio of $9: 1$. The mixture was degassed to remove any bubble, poured over the master, and then degassed again to ensure that all entrapped gases were eliminated. After baking and cooling, the PDMS was easily peeled and cut, and joined to the SAW device by pressure, thus forming microchannels without leakages that are the channels that conduct the gases to be measured. These microchannels have a height of $150 \mu \mathrm{m}$ and a width of $3.5 \mathrm{~mm}$ (Figure 3).

The SAW sensor is very sensitive to temperature; therefore, a Peltier device controlled by a PID program was used to keep the temperature constant. The glass transition temperature for PVP polymer is around $40^{\circ} \mathrm{C}$; therefore, the chosen temperature was $25^{\circ} \mathrm{C}$, which is suitable for maintaining the polymer in a rubbery state.

The sensor was tested using different concentrations of toluene. A computerized gas calibration system was used to vary the concentration of toluene in synthetic air. This was achieved by using mass flow controllers that provide desired 


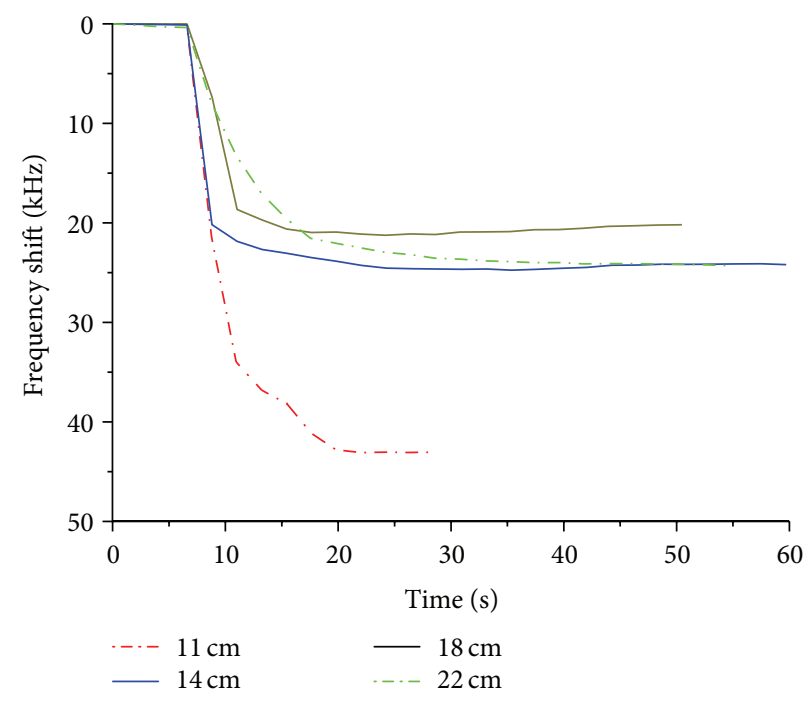

(a)

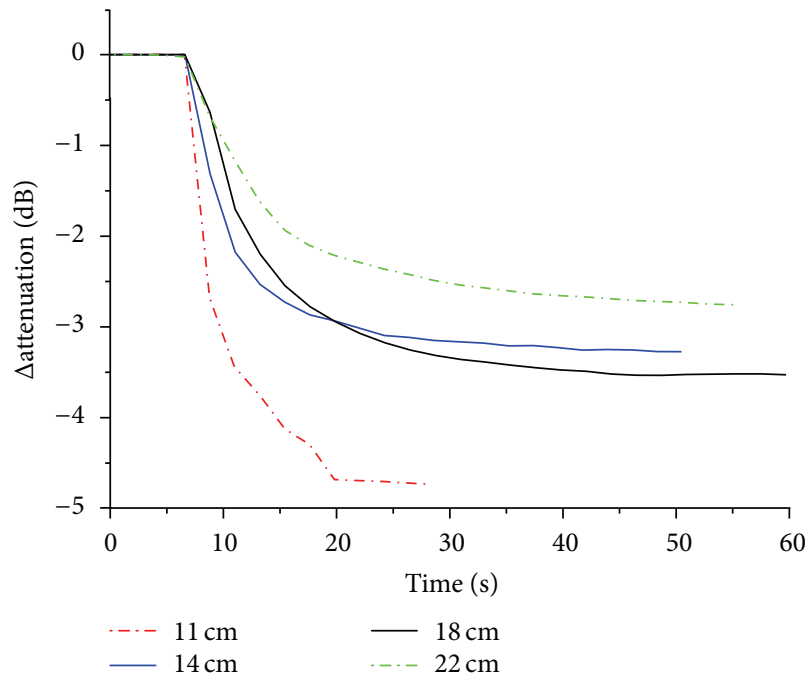

(b)

FIGURE 5: Frequency shift (a) and increasing of the attenuation (b) of the SAW sensor measured in real time due to the PVP electrospun fibers deposited for several needle-to-collector distances and an applied voltage of $18 \mathrm{kV}$.

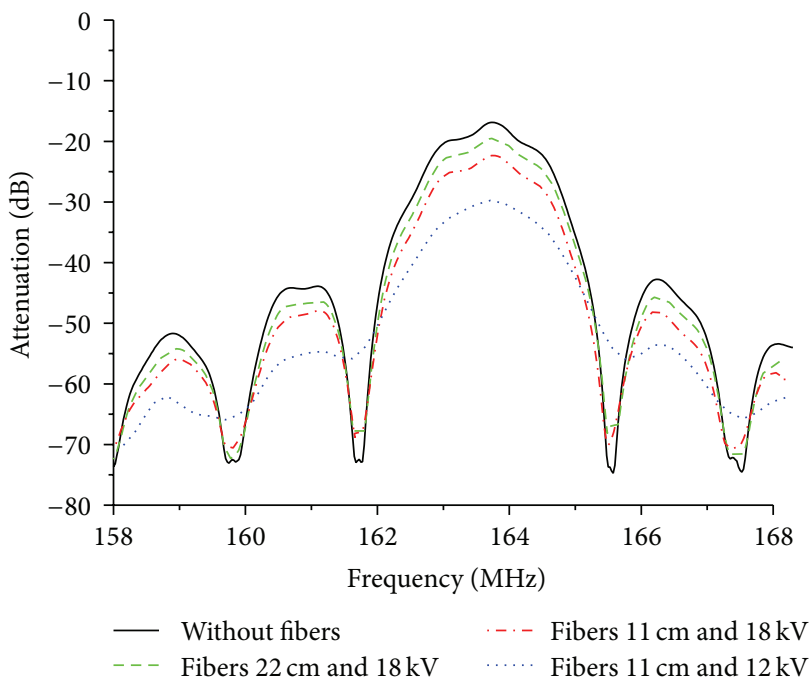

(a)

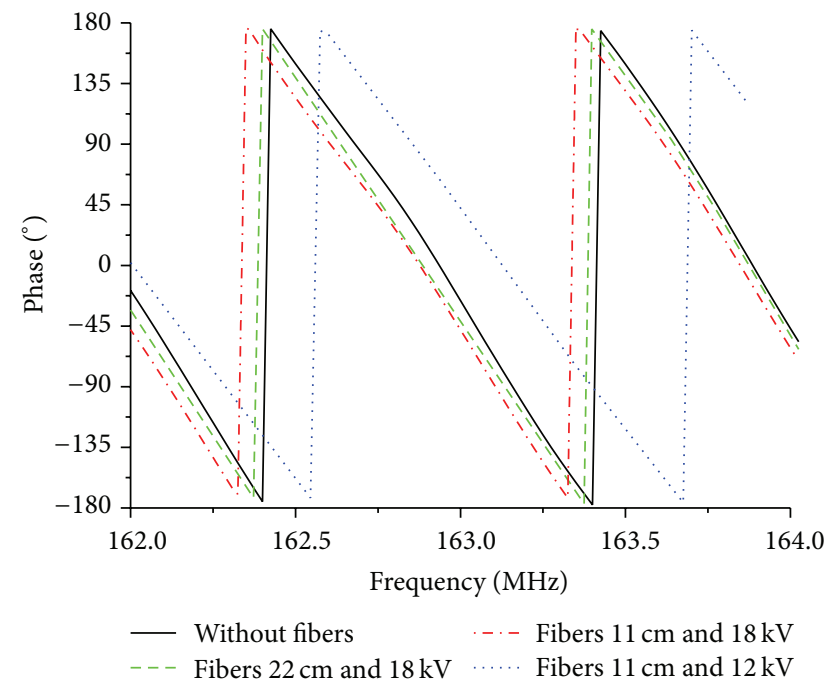

(b)

FIGURE 6: Amplitude (a) and phase shift (b) of the frequency response of the SAW device measured in the vector network analyzer with several combinations of applied voltage and needle-to-collector distance compared with the case of the bare SAW device.

concentrations, which were between $50 \mathrm{ppm}$ and $273 \mathrm{ppm}$. The total constant flow of the gas was kept at $10 \mathrm{~mL} \cdot \mathrm{min}^{-1}$ and the exposition and the purge times were 10 minutes. The responses were displayed in real time and saved for processing and analyzing.

\section{Results and Discussion}

The electrospun fibers were characterized in relation to the electric field intensity between the needle and the collector, which depends on the applied voltage and the needle-tocollector distance. The electrospinning was carried out with a constant distance between the needle and the collector of $11 \mathrm{~cm}$. Three different values of voltage were applied because different potentials lead to different electric fields affecting diameter and quantity of fibers in the device and therefore producing different frequency shifts and attenuations of the oscillating frequency of the Love-wave device. Then voltages of $12 \mathrm{kV}, 18 \mathrm{kV}$, and $19.5 \mathrm{kV}$ were applied and each one produced a different frequency shift (Figure 4(a)) and increase of the attenuation, calculated through (1) (Figure 4(b)) in the SAW sensor. These figures show that the fibers cause greater frequency shift and attenuation in the case of the lowest applied voltage. They also show that the detection of fibers 


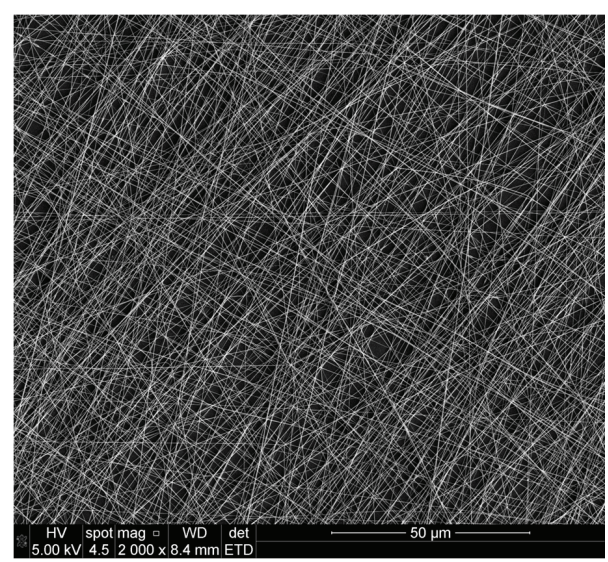

(a)

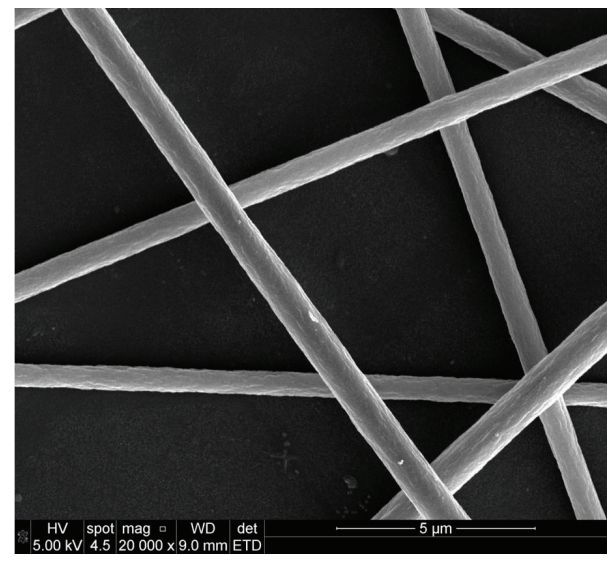

(b)

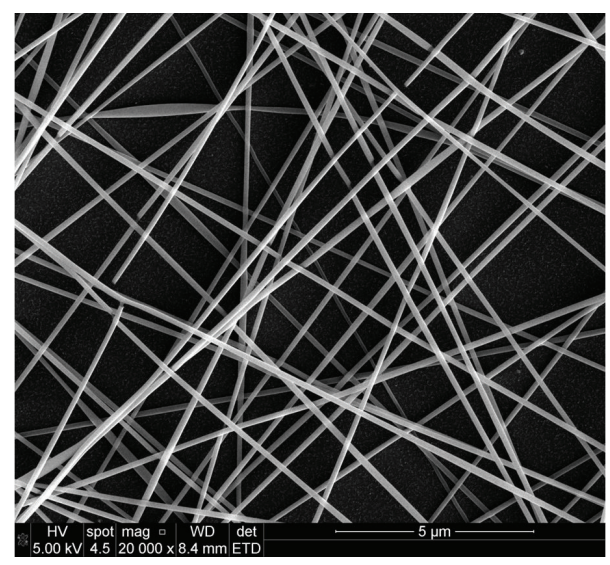

(c)

FIgURE 7: SEM images of the electrospun PVP fibers. (a) For $18 \mathrm{kV}$ and $22 \mathrm{~cm}$ of needle-to-collector distance. (b) For $18 \mathrm{kV}$ and $11 \mathrm{~cm}$ of needle-to-collector distance. (c) Detailed view of (a) image.

deposited on the SAW surface stops after about 15 seconds, although the fiber deposition continues. This fact means that the SAW sensor only detects the fibers deposited until that moment because the wave can only penetrate this first part of the layer. Based on the above information, we can say that the increase of the thickness of a fiber layer does not improve the detection of the VOC due to the short depth of penetration of mechanical perturbation into the fiber layer. Moreover, when $12 \mathrm{kV}$ was used, the increase of the attenuation caused by the fibers deposited is about $18 \mathrm{~dB}$ (see Figure 4(b)), which makes the device unsuitable to use as gas sensor. Therefore the highest voltages mentioned must be applied.

Figures 4(a) and 4(b) show that the electrospun fibers with $18 \mathrm{kV}$ and $19.5 \mathrm{kV}$ in the upper limit of our high voltage source give similar results. After that we studied the influence of the distance between the needle and the collector, while keeping the voltage at $18 \mathrm{kV}$. The frequency shift (Figure 5(a)) and the increase of the attenuation calculated through (1) (Figure 5(b)) of the SAW sensor were higher for the shortest needle-to-collector distance. Therefore, the longest distance must be chosen in order to obtain a layer of fibers to produce smaller losses. So, in this study it can be concluded that the most suitable combination of voltage and distance between the needle and the collector is $18 \mathrm{kV}$ and $22 \mathrm{~cm}$, respectively. These values will be finally used for the fiber-based sensor.

The frequency response of the SAW sensor was also measured by means of a network analyzer before and after the electrospinning process, the results being similar to those obtained in real time during the electrospinning process. Figures 6(a) and 6(b) show a comparison of the amplitude and phase of the frequency response of SAW devices prepared with different electrospinning conditions and one device without fibers. It can be seen in Figure 6(a) that the blue curve, corresponding to the lowest voltage, shows that the losses of the device are greater than $-32 \mathrm{~dB}$, what makes it unsuitable for sensing purposes, as we saw in the real-time measurement. It can be seen from Figure 6(a) that the best suited device for sensing purposes is the corresponding to $22 \mathrm{~cm}$ and $18 \mathrm{kV}$, as it was concluded before.

The fibrous films were examined by SEM and it was observed that longer distances and higher voltages caused smaller diameter in the electrospun fibers. Figure 7 shows images of the PVP electrospun fibers for $18 \mathrm{kV}$ applied voltage and distances of $11 \mathrm{~cm}$ and $22 \mathrm{~cm}$. In the RF measurements (Figure 6(a)) the losses are smaller for a distance needle to collector of $22 \mathrm{~cm}$ than for $11 \mathrm{~cm}$, but this difference was not very important. However, through the SEM images, it is observed that the needle-to-collector distance has an obvious impact on the morphology of fibers. The fiber diameters are greater when the needle-to-collector distance is $11 \mathrm{~cm}$ (Figure 7(b)). It can be seen in the figures that for this distance the diameters of the fibers are greater than $400 \mathrm{~nm}$, while for distance of $22 \mathrm{~cm}$ (Figure 7 (c)) the diameters are smaller than $120 \mathrm{~nm}$. Figure 7(a) shows a top view of the surface of the device at a magnification $2000 \mathrm{x}$, appearing like a $3 \mathrm{D}$ nanofiber film tissue. Structure thickness varied between 1.5 and $5 \mu \mathrm{m}$. From the above results, greater frequency shifts in the detection of VOCs for devices with sensitive layers of nanofibers with lower diameters are expected due to the larger active surface of the nanofibers layer, since larger surface enhances VOCs' adsorption and then improves the sensitivity and reaction rates. It is very important to have a system that is capable of estimating the amount of fibers deposited on the SAW device, which is closely related to the $\mathrm{RF}$ losses. The system presented in this work is capable of 


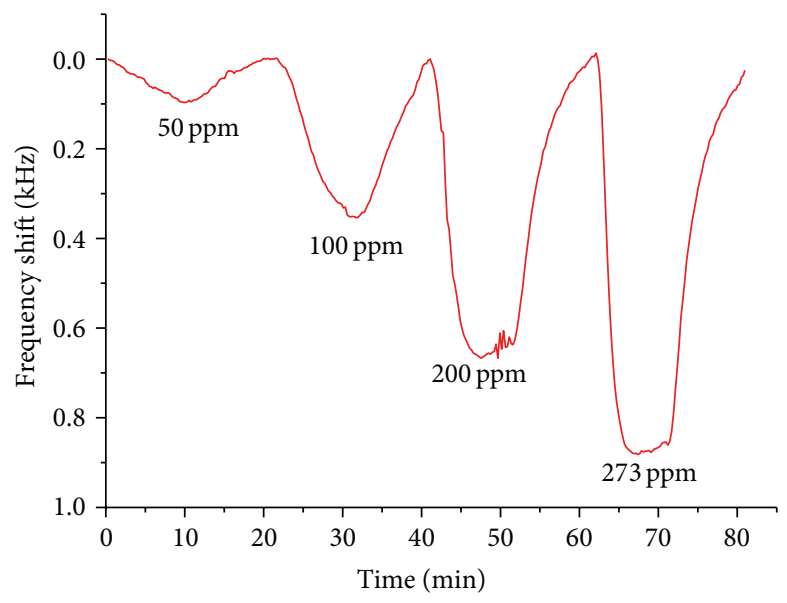

(a)

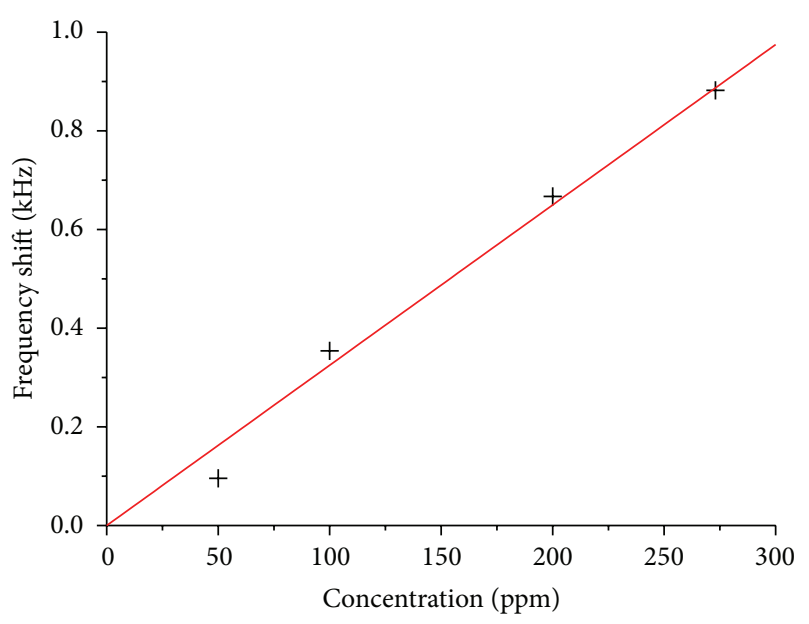

(b)

FIGURE 8: (a) Frequency shift in real time of the SAW sensor with a sensitive layer of electrospun PVP nanofibers for different concentrations of toluene and (b) the linear relation between frequency shift and the concentration.

determining the RF losses in the SAW device in real time and therefore allows the selection of the optimum amount of fibers deposited in order to achieve maximum sensitivity of the device in real time.

The gas sensing properties of the PVP nanofibers on the surface of the SAW device were investigated. The device included two DLs, one used as reference and the other one as a sensor with a sensitive layer of electrospun PVP nanofibers. The difference between the frequency shifts of both DLs was used to compensate for common error sources in the measurement, such as changes in pressure, temperature, and flow. Four different concentrations of toluene were measured in synthetic air (50 ppm, $100 \mathrm{ppm}, 200 \mathrm{ppm}$, and $273 \mathrm{ppm}$ ). After each exposure, the sensing chamber was purged with synthetic air, shifting the frequency back to baseline. As concentrations of toluene were increased, the frequency shift rose gradually, as it can be seen in Figure 8(a). Also different exposures to the same concentration of toluene were tested. The frequency shift showed very good repeatability. The maximum frequency shift for each concentration was taken as input data for the correlation with the concentration, which showed a good linear relationship (Figure 8(b)).

In our system the minimum frequency shift which can be clearly detected is $30 \mathrm{~Hz}$. The slope of the fit in Figure $8(\mathrm{~b})$ shows that the sensitivity was $3.25 \mathrm{~Hz} / \mathrm{ppm}$. This leads to a detection limit of about $10 \mathrm{ppm}$. The only work we have found that deals with toluene detection with nanofiber-based sensors is one with a SAW sensor with PEO nanofibers [26]. In that work the sensitivity is $0.45 \mathrm{~Hz} / \mathrm{ppm}$. Nicolae et al. [30] reported sensitivities to toluene with SAW sensors with polyethylenimine-based films and nanocomposites between 1.2 and $5.4 \mathrm{~Hz} / \mathrm{ppm}$.

\section{Conclusions}

Electrospinning technique is an efficient method of depositing polymers in the form of micro- and nanofibers. This research presents performances of the SAW sensor with PVP electrospun nanofibers as a sensitive layer. The diameter of the fibers increases with a decrease in both the applied voltage and the distance between the needle and the collector. By characterizing the electrospinning process of the fibers in real time, it has been demonstrated that the frequency does not change from a given amount of fibers deposited. This could be due to the fact that the waves penetrate only the first few layers of deposited fibers. Therefore, we can conclude that the VOCs adsorbed by the more external fibers cannot be detected by the sensor. Thus, these fibers do not improve the sensitivity of the device. In our electrospinning configuration, using a solution of PVP in deionized $\mathrm{H}_{2} \mathrm{O} 25 \%$ wt with an applied voltage of $18 \mathrm{kV}$ and a needle-to-collector distance of $22 \mathrm{~cm}$, the optimal nanofiber deposition time was about 15 seconds. For longer durations, the frequency shift and the attenuation were constant. The fibrous film obtained with these parameters analyzed by SEM showed nanofibers with diameters lower than $120 \mathrm{~nm}$ which enhances the absorption of gas.

In order to get the optimum amount of deposited fiber, the process has been monitored in real time by measuring the feeding current of the amplifying device by means of an amperimeter. This allowed the estimation of the increase in RF losses of the SAW device using a linear relationship.

The polymer fibers are suitable to use as a sensitive layer on SAW devices in order to detect VOCs. Sensors' response to toluene exhibited a linear relationship in the 0 $273 \mathrm{ppm}$ range. This study demonstrated a method of efficient detection using electrospun PVP nanofibers deposited on SAW devices.

\section{Conflict of Interests}

The authors declare that there is no conflict of interests regarding the publication of this paper. 


\section{Acknowledgments}

This work has been supported by the Spanish Science and Innovation Ministry under the Project TEC2010-21357-C0504. The authors want to thank University of Extremadura for SEM analysis.

\section{References}

[1] G. A. Eiceman and Z. Karpas, Mobility Spectrometry, CRC Press, 2nd edition, 2005.

[2] R. Cumeras, I. Gràcia, E. Figueras et al., "Finite-element analysis of a miniaturized ion mobility spectrometer for security applications," Sensors and Actuators B: Chemical, vol. 170, pp. 13-20, 2012.

[3] T. Logan, E. Allen, M. Way, A. Swift, S.-D. Soni, and I. Koplovitz, "A method for the analysis of tabun in multisol using gas chromatographic flame photometric detection," Toxicology Mechanisms and Methods, vol. 16, no. 7, pp. 359-363, 2006.

[4] M. E. Webber, M. Pushkarsky, and C. K. N. Patel, "Optical detection of chemical warfare agents and toxic industrial chemicals: simulation," Journal of Applied Physics, vol. 97, no. 11, Article ID 113101, pp. 1-11, 2005.

[5] F. Yan and T. Vo-Dinh, "Surface-enhanced Raman scattering detection of chemical and biological agents using a portable Raman integrated tunable sensor," Sensors and Actuators B: Chemical, vol. 121, no. 1, pp. 61-66, 2007.

[6] K. Song, B. Ahn, E. Jung, Y.-I. Lee, and S. Ko, "Enhancement of the detection sensitivity for volatile organic compounds by using an annular type photoionization detector and a preconcentration system," Analytica Chimica Acta, vol. 583, no. 1, pp. 210-215, 2007.

[7] Z. X. Cheng, X. H. Ren, J. Q. Xu, and Q. Y. Pan, "Mesoporous $\mathrm{In}_{2} \mathrm{O}_{3}$ : effect of material structure on the gas sensing," Journal of Nanomaterials, vol. 2011, Article ID 654715, 6 pages, 2011.

[8] L. A. Pinnaduwage, T. Thundat, J. E. Hawk et al., "Detection of 2,4-dinitrotoluene using microcantilever sensors," Sensors and Actuators B: Chemical, vol. 99, no. 2-3, pp. 223-229, 2004.

[9] C. Karnati, H. Du, H.-F. Ji et al., “Organophosphorus hydrolase multilayer modified microcantilevers for organophosphorus detection," Biosensors and Bioelectronics, vol. 22, no. 11, pp. 2636-2642, 2007.

[10] J. Amírola, A. Rodríguez, L. Castañer, J. P. Santos, J. Gutiérrez, and M. C. Horrillo, "Micromachined silicon microcantilevers for gas sensing applications with capacitive read-out," Sensors and Actuators B: Chemical, vol. 111-112, pp. 247-253, 2005.

[11] D. Matatagui, M. J. Fernández, J. Fontecha et al., "Lovewave sensor array to detect, discriminate and classify chemical warfare agent simulants," Sensors and Actuators B: Chemical, vol. 175, pp. 173-178, 2012

[12] N.-J. Choi, J.-H. Kwak, Y.-T. Lim et al., "Classification of chemical warfare agents using thick film gas sensor array," Sensors and Actuators B: Chemical, vol. 108, no. 1-2, pp. 298-304, 2005.

[13] I. Castro-Hurtado, J. Herrán, G. Mandayo, and E. Castaño, " $\mathrm{SnO}_{2}$-nanowires grown by catalytic oxidation of tin sputtered thin films for formaldehyde detection," Thin Solid Films, vol. 520, no. 14, pp. 4792-4796, 2012.

[14] L. Blanc, A. Tetelin, C. Boissière, G. Tortissier, C. Dejous, and D. Rebière, "Love wave characterization of the shear modulus variations of mesoporous sensitive films during vapor sorption," IEEE Sensors Journal, vol. 12, no. 5, pp. 1442-1449, 2012.
[15] M. C. Horrillo, J. Martí, D. Matatagui et al., "Single-walled carbon nanotube microsensors for nerve agent simulant detection," Sensors and Actuators B: Chemical, vol. 157, no. 1, pp. 253-259, 2011.

[16] D. N. Chavan, G. E. Patil, D. D. Kajale, V. B. Gaikwad, P. K. Khanna, and G. H. Jain, "Nano Ag-doped $\operatorname{In}_{2} \mathrm{O}_{3}$ thick film: a low-temperature $\mathrm{H}_{2} \mathrm{~S}$ gas sensor," Journal of Sensors, vol. 2011, Article ID 824215, 8 pages, 2011.

[17] F. Bender, N. Barié, G. Romoudis, A. Voigt, and M. Rapp, "Development of a preconcentration unit for a SAW sensor micro array and its use for indoor air quality monitoring," Sensors and Actuators B: Chemical, vol. 93, no. 1-3, pp. 135-141, 2003.

[18] D. S. Ballantine, R. M. White, S. J. Martin et al., Acoustic Wave Sensors-Theory, Design, and Physico-Chemical Applications, Academic Press, New York, NY, USA, 1997.

[19] J. W. Grate, S. J. Martin, and R. M. White, "Acoustic wave microsensors: part I," Analytical Chemistry, vol. 65, no. 21, pp. 940-948, 1993.

[20] H. Liu, X. Ding, G. Zhou, P. Li, X. Wei, and Y. Fan, "Electrospinning of nanofibers for tissue engineering applications," Journal of Nanomaterials, vol. 2013, Article ID 495708, 11 pages, 2013.

[21] J. Doshi and D. H. Reneker, "Electrospinning process and applications of electrospun fibers," Journal of Electrostatics, vol. 35, no. 2-3, pp. 151-160, 1995.

[22] A. Urrutia, J. Goicoechea, P. J. Rivero, I. R. Matías, and F. J. Arregui, "Electrospun nanofiber mats for evanescent optical fiber sensors," Sensors and Actuators B: Chemical, vol. 176, pp. 569-576, 2013.

[23] X. Song and L. Liu, "Characterization of electrospun ZnO$\mathrm{SnO}_{2}$ nanofibers for ethanol sensor," Sensors and Actuators A: Physical, vol. 154, no. 1, pp. 175-179, 2009.

[24] W. S. Khan, R. Asmatulu, and M. M. Eltabey, "Electrical and thermal characterization of electrospun PVP nanocomposite fibers," Journal of Nanomaterials, vol. 2013, Article ID 160931, 9 pages, 2013.

[25] L. Sheng, C. Dajing, and C. Yuquan, "A surface acoustic wave humidity sensor with high sensitivity based on electrospun MWCNT/Nafion nanofiber films," Nanotechnology, vol. 22, no. 26, Article ID 265504, 2011.

[26] S. Liu, H. Sun, R. Nagarajan et al., "Dynamic chemical vapor sensing with nanofibrous film based surface acoustic wave sensors," Sensors and Actuators A: Physical, vol. 167, no. 1, pp. 8-13, 2011.

[27] X. He, R. Arsat, A. Z. Sadek, W. Wlodarski, K. Kalantar-zadeh, and J. Li, "Electrospun PVP fibers and gas sensing properties of PVP/36。 YX $\mathrm{LiTaO}_{3}$ SAW device," Sensors and Actuators B: Chemical, vol. 145, no. 2, pp. 674-679, 2010.

[28] Q. Lin, Y. Li, and M. Yang, "Highly sensitive and ultrafast response surface acoustic wave humidity sensor based on electrospun polyaniline/poly(vinyl butyral) nanofibers," Analytica Chimica Acta, vol. 748, pp. 73-80, 2012.

[29] D. Matatagui, Development of gravimetric sensors to detect chemical and biological warfare agents [Ph.D. thesis], Universidad Autónoma de Madrid, 2012.

[30] I. Nicolae, C. Viespe, and C. Grigoriu, "Nanocomposite sensitive polymeric films for SAW sensors deposited by the MAPLE direct write technique," Sensors and Actuators B: Chemical, vol. 158, no. 1, pp. 418-422, 2011. 

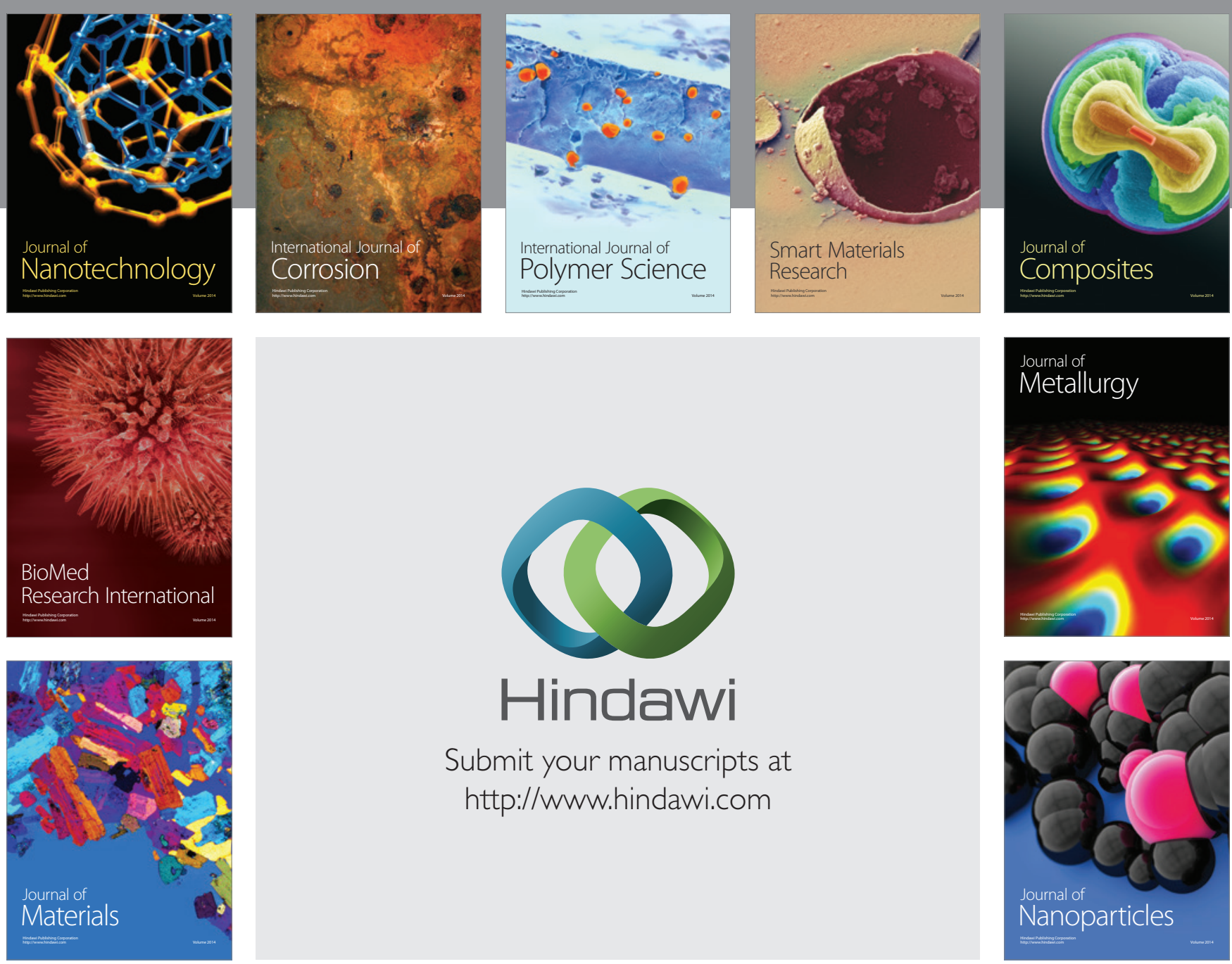

Submit your manuscripts at http://www.hindawi.com
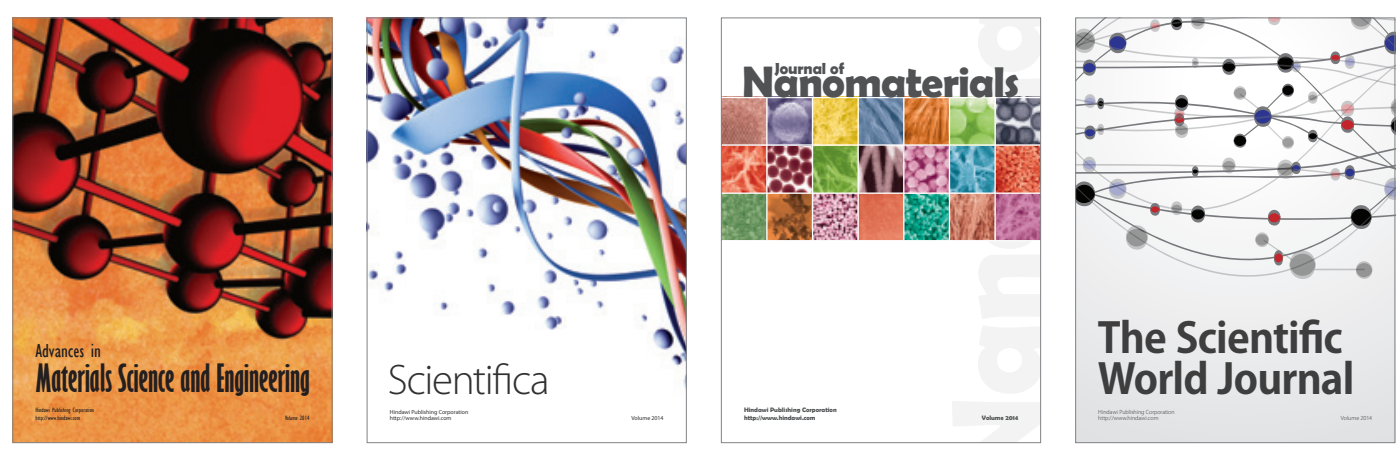

\section{The Scientific World Journal}
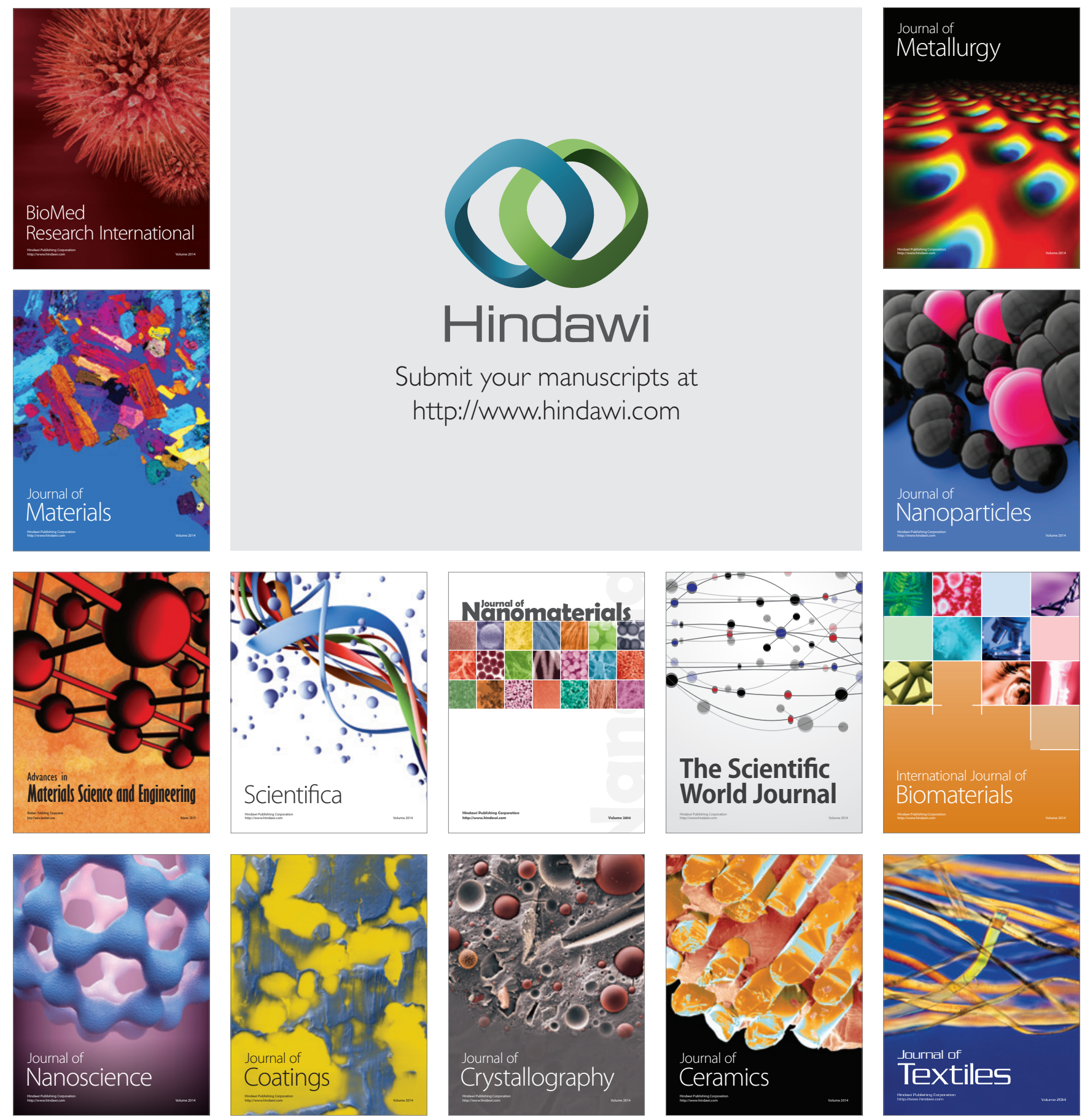